\title{
Gestion des périmètres d'irrigation en temps réel en face d'une sécheresse
}

\author{
M. Jean, A. Galand \\ Société du Canal de Provence
}

\section{Agriculture irriguée et sécheresse estivale}

En agriculture irriguée, un certain niveau de sécheresse (déficit hydrique) est tolérable : la production des cultures vis-à-vis de l'eau utilisée suit une loi de rendements décroissants.

Il ne serait donc pas réaliste de concevoir des moyens d'irrigation (ouvrages, matériels, main-d'œuvre) capables de satisfaire l'intégralité des besoins, y compris les pointes tout à fait exceptionnelles. On peut même ajouter que pour certaines cultures (en particulier les productions de semence en périodes végétatives) la satisfaction des besoins en eau au niveau des consommations potentielles (évapotranspiration maximale ETM) ne permet pas d'atteindre les meilleurs rendements.

Cependant, pour de nombreuses cultures, il existe des périodes pendant lesquelles toute restriction au-dessous d'un certain seuil entraîne une perte de potentiel de production irrécupérable quelle que soit l'alimentation hydrique ultérieure.

Ces périodes critiques dépendent de la culture et de son stade végétatif (par exemple le maïs est beaucoup plus sensible à certaines sécheresses temporaires que le soja et surtout que le tournesol et le sorgho). Elles doivent donc être prises en compte par l'exploitant agricole s'il doit, à son niveau, gérer la pénurie.

Il en résulte donc que l'impact économique d'une pénurie peut, dans une certaine mesure, être atténué selon le degré d'information et la technicité de l'exploitant agricole.
On ne peut cependant ignorer qu'au cours des dernières décennies, les progrès techniques acquis sur les autres facteurs de production (génétique, engrais, produits phytosanitaires) ont considérablement augmenté les performances attendues de la culture irriguée. Il en résulte que l'effet limitant du facteur eau d'irrigation a aujourd'hui des conséquences plus graves qu'autrefois sur l'économie des exploitations.

\section{Les irrigations de Basse Durance au début du $20^{\mathrm{e}}$ siècle}

La plaine de la Basse Durance, en aval du confluent avec le Verdon, est une zone d'agriculture irriguée intensive dépendant d'ouvrages collectifs de transport et de distribution plusieurs fois séculaires.

Antérieurement à l'aménagement de la Durance entrepris par Electricité de France dans le cadre de la loi du 5 janvier 1955, les différents périmètres d'irrigation étaient alimentés à partir de prises directes en Durance s'échelonnant entre Pont Mirabeau (au confluent du Verdon) et le Rhône.

Ces irrigations étaient soumises à de sévères pénuries : le débit disponible pouvait descendre à des valeurs voisines de $40 \mathrm{~m}^{3} / \mathrm{s}$ à l'aval de Pont Mirabeau, alors que la somme des prélèvements possibles pour la basse vallée était de l'ordre de $85 \mathrm{~m}^{3} / \mathrm{s}$.

\section{Management of irrigation perimeters in real time when faced with a drought}

The regional context of hydro-agricultural facilities in Basse Durance and its development during the 20th century is an interesting reference. Before the Durance-Verdon group was regularized, the irrigated perimeters served underwent serious shortages. This report will describe the system used to manage the shortage set up at the beginning of the century to enable available water to be better divided and thus avoid serious conflicts between holders of endowments. Agricultural reserves made up today have profoundly changed the situation and have been sufficient to avoid the return of any serious shortages. 


\section{Commission des prises d'eau de la Basse Durance}

Textes de création

- Loi du 11 juillet 1907 sur la réglementation des eaux de la Durance.

— Décret du 14 août 1908 portant règlement d'administration publique pour l'exécution de la loi ci-dessus.

\section{Fonctions}

Assurer la répartition en situation de pénurie des débits disponibles en Durance (à l'aval du pont Mirabeau) entre les prises concédées ou à concéder.

\section{Composition}

15 membres:

- 5 membres élus par les concessionnaires du département du Vaucluse ;

- 5 membres élus par les concessionnaires du département des Bouches-du-Rhône;

- 5 membres nommés par le Ministère de l'agriculture.

Fonctionnement

- Un Directeur élu par la commission et un Directeur Adjoint nommé par le Ministre de l'Agriculture ;

- la commission exécutive se ré unit "toutes les fois que les besoins du service l'exigent ".

\section{La commission des prises d'eau de la Basse Durance}

Les inévitables conflits entrainés par ces situations fréquentes de pénurie étaient gérés par une commission mise en place par le législateur. Cette commission, qui se réunissait " chaque fois que les besoins du service l'exigeaient ", avait pour fonction de répartir les disponibilités entre les ayants droit.

\section{Les critère de répartition de l'eau entre concessionnaires}

La législation précisait les critères de gestion qui étaient à mettre en œuvre par la commission.

Le débit de la Durance mesuré aux environs du viaduc de Barbentane, soit en aval des zones de prélèvement, constituait le paramètre de décision. Deux seuils de débit avaient été définis : un seuil à $6 \mathrm{~m}^{3} / \mathrm{s}$ («premier minimum ») et un deuxième seuil à $2 \mathrm{~m}^{3} / \mathrm{s}$ ("second minimum $")$.

Lorsque le débit constaté à Barbentane était supérieur " au premier minimum », les prélèvements étaient entièrement libres. Lorsque le débit aval diminuait pour atteindre le seuil du "premier minimum ", chaque concessionnaire de dotation devait limiter le débit de sa prise à la « dotation réglementaire ". Un effort supplémentaire était demandé lorsque le "second minimum " de débit était atteint, tous les concessionnaires devant successivement réduire leur prélèvement «à la quantité strictement nécessaire pour les besoins à desservir ". Si le débit résiduel venait encore à descendre en dessous de ce " second minimum ", des mesures de réduction des prélèvements plus sévères étaient alors imposées par le directeur de la commission exécutive.

Ces règles de gestion, qui s'appliquaient à une quinzaine de prises en Durance, étaient d'une application délicate, car la loi précisait que les mesures prescrites devaient être appliquées en commençant par les concessions les plus récentes.

En outre, la priorité donnée à l'alimentation en eau des agglomérations était déjà affirmée : parmi les concessionnaires, le canal de Marseille bénéficiait d'un plancher minimum garanti $\left(5,5 \mathrm{~m}^{3} / \mathrm{s}\right)$ "dans l'intérêt de la santé publique $"$.

\section{Les ouvrages de réglage des débits}

Les ouvrages mis alors en place pour assurer la répartition des débits entre les différents concessionnaires matérialisent cette réglementation. Ainsi pour l'ancien partiteur du Merle, situé sur le canal du congrès au voisinage de Salon de Provence. Cet ouvrage répartit le débit disponible avec un réglage par déversoirs dont les largeurs sont proportionnelles aux dotations respectives de chaque concessionnaire. En outre, les cotes de calage de ces déversoirs sont décalées en altitude, de manière à réaliser automatiquement une priorité aux concessions les plus anciennes.

\section{Gestion de la pénurie au sein d'un périmètre irrigué}

A un niveau inférieur du système hydraulique, les « rationnements " ainsi réalisés en période de pénurie doivent être répartis au sein des réseaux d'irrigation jusqu'aux utilisateurs. Selon quelles modalités? L'exemple du canal de Carpentras présente parfaitement le problème à travers un texte datant de 1925 (Le canal de Carpentras par Robert CAILlot) :

"Au canal de Carpentras, dès que les ordres de réduction arrivent, le service de l'exploitation prend des mesures immédiates pour faire opérer sur les filioles les mêmes réductions.

Si pendant six mois sur huit, le module attribué à chaque filiole est de 1,20 litre par hectare et par seconde, en période de pénurie (deux mois environ) ce module s'abaisse à 11 , $0,901,0,801,0,701,0,601$ et quelquefois même 0,501 par hectare et par seconde.

Bien que le règlement sur le service des arrosages donne au service de l'exploitation le droit de maintenir, malgré les pénuries, un volume normal dans les filioles, mais en réduisant alors le nombre de filioles ouvertes à la fois dans chaque section, il n'est pas fait application de cette mesure.

Nous avons la conviction que l'application de cette faculté soulèverait de véhémentes protestations et que le "pis aller ", parfaitement accepté d'ailleurs par les arrosants en temps de pénurie, consiste à faire porter la réduction sur le volume introduit dans les filioles. ”

On aura compris que le rédacteur utilisait le terme de volume pour celui de débit. 


\section{Commission de gestion des réserves}

Dénomination exacte

Commission de gestion de la réserve agricole de SerrePonçon et des réserves du Verdon.

Textes de création

- Loi du 5 janvier 1955 relative à l'aménagement de la Durance et convention entre le Ministre de l'Agriculture et Electricité de France du 24 novembre 1953 y annexée.

- Arrêté du Ministre de l'Agriculture du 10 septembre 1970.

Fonctions

Prendre les dispositions nécessaires pour gérer les réserves agricoles de Serre-Ponçon et du Verdon (cf. art. 7 de l'arrêté du 10 septembre 1970).

\section{Composition}

- Président : Préfet de la Région Provence-Alpes-Côte d'Azur.

- I $I^{e r}$ Vice-Président: Ingénieur Général du GREF chargé de la Région Provence-Alpes-Côte d'Azur.
- $2^{e}$ Vice-Président : Ingénieur Général des Ponts \& Chaussées, chargé de la $33^{e}$ circonscription des Services de la navigation.

- 12 représentants des usagers (dont 6 désignés par la Commission exécutive de la Durance).

- 11 fonctionnaires (DDA-DDE-SRAE-DRIR).

Secrétariat

Le secrétariat de la Commission est assuré par le Service régional de l'aménagement des eaux de la Région Provence-Alpes-Côte d'Azur.

Coût de fonctionnement

La Commission n'a pas de budget. Les frais de secrétariat sont à la charge du SRAE. Les frais de déplacement des différents fonctionnaires sont à la charge de leurs administrations respectives.

Périodicité

Réunion une fois à Marseille avant le 15 avril et chaque fois que les circonstances l'exigent.

\section{Situation actuelle des aménagements de la Basse Durance}

L'état actuel de fonctionnement de ce système résulte de l'aménagement de la Durance entrepris par EDF (Electricité de France) dans le cadre de la loi du 5 janvier 1955.

Il s'agit d'un aménagement d'ensemble, hydroélectrique et hydroagricole, avec une régulation de la Durance obtenue grâce à l'édification du barrage de Serre-Ponçon. Un accord passé par le Ministère de l'Agriculture prévoit qu'EDF fournira aux irrigants de Basse Durance un débit journalier égal à celui qui passerait à Pont Mirabeau si Serre-Ponçon n'existait pas. De plus, cette retenue comporte une réserve d'eau de $200 \mathrm{Mm}^{3}$ disponible pour l'Agriculture pendant les 3 mois d'été (juillet, août, septembre).

La gestion de cette réserve agricole est assurée par une commission créée à cet effet.

- du $1^{\text {er }}$ au 30 juin, il n'y a en principe pas de déstockage possible. Il faudra donc se contenter du débit naturel de la Durance reconstitué à Cadarache. Au cas où ce débit serait insuffisant, il faudra que la Commission Exécutive de la Durance instaure une réduction collective des prélèvements étant entendu qu'une priorité de desserte est accordée à la satisfaction des besoins en eau potable ;

- à partir du $1^{\text {er }}$ juillet et jusqu'au 30 septembre le déstockage peut intervenir, et s'effectuer selon le principe suivant :

L'agriculture demande en début de saison que soit distribué à Pont Mirabeau un certain débit pour l'irrigation en Basse Durance (en général fixé à $107 \mathrm{~m}^{3} / \mathrm{s}$ en juillet-août et $91,5 \mathrm{~m}^{3} / \mathrm{s}$ en septembre). EDF doit calculer jour après jour le débit naturel de la Durance (celui qui passerait à Pont Mirabeau si les barrages amont n'existaient pas), sommer journellement les différences (débit fourni - débit naturel) en ne comptabilisant que les termes positifs, et en déduire le volume à déstocker pour l'agriculture.

L'objectif visé par l'Agriculture est de fournir aux agriculteurs un débit suffisant pour l'irrigation, tout en ne dépassant pas le volume de réserve disponible de $200 \mathrm{Mm}^{3}$. 
On doit donc déterminer en début de saison la position du seuil de débit à délivrer.

Dans le cas où la tendance de l'année serait de dépasser les $200 \mathrm{Mm}^{3}$ alloués, il faut alors mettre en place une restriction volontaire du débit journalier demandé par l'agriculture.

Le problème qui se pose est donc de présumer avant le $1^{\text {er }}$ juillet si l'on est en année défavorable, et si oui de déterminer quels doivent être semaine après semaine les seuils à imposer pour éviter le dépassement du volume total de $200 \mathrm{Mm}^{3}$.

Ce problème est donc lié à la prévision des étiages. On doit chercher à déterminer en début de saison, en fonction des pluies d'hiver, de printemps, des pluies et débits en mai, juin, des températures, etc., quel volume déstocké on est susceptible d'atteindre au 30 septembre et, si ce volume dépasse la limite, quelles restrictions on doit mettre en place (ces restrictions pouvant être "actualisées " à tout moment au cours de l'été).

C'est sur la base de ces orientations générales que le CEMAGREF et le SRAE Provence-Côte d'Azur ont élaboré en 1977 un modèle de prévision permettant tout au long de la saison estivale de fournir à la Commission exécutive des éléments de décision relatifs aux consignes de déstockage de la retenue à adopter.

En pratique, il faut cependant constater que l'effet régulateur de la réserve agricole semble dépasser les espoirs initiaux: alors qu'on ne prévoyait initialement qu'une satisfaction totale des besoins avec une fréquence de $80 \%$, on observe qu'aucune restriction n'a dû être imposée aux utilisateurs depuis la mise en service de Serre-Ponçon (1960). L'année 1989 sera peut-être la première année où la Commission exécutive mise en place aura véritablement des décisions effectives à prendre pour faire face à une situation de pénurie.

\section{Les aménagements dépendant du Verdon}

Les réserves agricoles constituées sur le Verdon (convention agriculture/EDF du 21 mai 1962) représentent un volume total de 250 millions de mètres cubes, comprenant :

- 85 millions de $\mathrm{m}^{3}$ derrière le barrage de Castillon ;

- 140 millions de $\mathrm{m}^{3}$ derrière le barrage de Sainte-Croix ;

- 25 millions de $\mathrm{m}^{3}$ stockés dans le réservoir de Bimont.

Cette réserve a été entièrement affectée à la Société du Canal de Provence par convention du 26 mai 1975, la Société étant quasiment le seul organisme prélevant de l'eau du Verdon.

La fonction assignée à ces réserves et le type d'ouvrages qu'elles desservent les distinguent de la réserve de la Durance sur plusieurs points qui rendent plus improbables les risques de pénurie :

- La réserve de $200 \mathrm{Mm}^{3}$ constituée sur la Durance est une réserve de régulation annuelle. Les volumes d'eau éventuellement non utilisés en fin de période sont remis à la disposition d'EDF qui les utilisera au mieux de ses intérêts.

Par contre les réserves du Verdon visent à une régulation interannuelle. Le stock résiduel en fin de saison estivale (16 octobre) n'est pas utilisable par EDF.

- Les ouvrages alimentés, desservis par le Canal de Provence, sont des ouvrages récents conçus selon des techniques modernes :

- les ouvrages de distribution sont constitués par des réseaux de canalisations enterrées et sous pression conçus pour la pratique de l'irrigation par aspersion et à la demande. Cette technique d'irrigation est beaucoup plus efficiente que les techniques anciennes d'irrigation de surface, si bien qu'à surface irriguée équivalente, on constate que les réseaux modernes sous pression prélèvent 3 à 4 fois moins d'eau que les anciens réseaux de surface (globalement, les aménagements récents dérivent en tête des ouvrages de transport 5 à 10 fois moins d'eau que les aménagements anciens) ;

- les ouvrages de transport (canaux et galeries) sont gérés par une régulation moderne ("régulation dynamique ») : une télégestion centralisée, pilotée en temps réel par un calculateur industriel, ajuste les débits transités en fonction de l'évolution prévue de la demande et de l'état des stocks dans le système hydraulique. Ces stocks ne sont pas négligeables $\left(1 \mathrm{Mm}^{3}\right.$ non compris les réserves de Bimont et du Trapan). Ils pourraient être valorisés pour optimiser les déstockages demandés sur les réserves du Verdon, dans le cas où les débits naturels reconstitués enregistreraient des variations d'amplitude limitée sur des périodes de quelques journées.

- Enfin ces ouvrages sont en cours de développement, si bien que les besoins actuels sont de l'ordre de 200 millions de mètres cubes (dont 100 entre le $1^{\text {er }}$ mars et le 30 septembre), alors que les volumes dérivables sont globalement fixés à $660 \mathrm{Mm}^{3}$.

Ces différents éléments concourent à placer les aménagements dépendant du Verdon dans une situation peu exposée à un risque de pénurie. Cependant, dans le cas de situations exceptionnelles qui pourraient se présenter dans l'avenir, les équipements mis en place pourraient être gérés de manière à imposer un rationnement : d'une part, au niveau des têtes de réseaux de distribution (prises d'eau sur les ouvrages principaux), les organes de réglage télécommandés depuis le Centre général de télécontrôle permettraient généralement de limiter les débits dérivés à des valeurs plafonds. D'autre part, au niveau de la distribution aux prises d'irrigation, les « conditions générales de fourniture des eaux " de la SCP ont prévu des situations exceptionnelles où la fourniture de l'eau "à la demande» pouvait être interrompue : "si le fonctionnement du réseau le nécessite, la Société se réserve le droit d'instituer un tour de rôle ou un service réduit pour assurer une desserte équitable des abonnés" ". 


\section{Conclusion}

Le contexte régional est historiquement marqué en Basse Durance par des pénuries graves et fréquentes. Le législateur avait su, à l'époque, créer les moyens (la Commission des prises d'eau de la Basse Durance) et énoncer les critères de gestion qui ont permis de limiter les inévitables conflits qui en résultaient.

L'aménagement Durance-Verdon a radicalement modifié cette situation.

En Basse Durance, les besoins sont maintenant entièrement satisfaits avec une probabilité sans doute nettement supérieure à $80 \%$. Il reste cependant que les modalités d'organisation des déstockages en année d'extrême sécheresse restent à mettre au point, en testant les modèles décisionnels qui ont été proposés.

Les aménagements dépendant du Verdon apparaissent dans une situation particulièrement favorable vis-à-vis du risque de pénurie : d'une part les réserves constituées assurent une régulation interannuelle, et d'autre part les ouvrages desservis sont des ouvrages modernes, économes en eau, et gérés par une régulation élaborée susceptible d'optimiser les déstockages à effectuer. 